\title{
Effects of Vitamin C and NADH on in Vitro Proliferative Function of Human Lymphocytes Exposed to Pesticides (Mancozeb and Metribuzin)
}

\author{
Amel Medjdoub ${ }^{1}$, Amel Saidi Merzouk ${ }^{1}$, Hafida Merzouk ${ }^{1}$ and Ilyes Baghli ${ }^{2}$ \\ 1. Laboratory of Physiology, Physiopathology and Biochemistry of Nutrition, Department of Biology, Faculty of Natural and Life
} Sciences, Earth and Universe, University ABOU-BEKR BELKAÏD, Tlemcen 13000, Algeria

2. Society of Nutrition and Orthomolecular Medicine, SANMO, Algeria.

\begin{abstract}
Antioxidants such as vitamin $\mathrm{C}$ and $\mathrm{NADH}$ can protect against pesticide adverse effects on immune function. The aim of this work was to study the in vitro effects of vitamin $\mathrm{C}$ and NADH on the proliferative responses of human lymphocytes exposed to pesticides (Mancozeb fungicide and Metribuzin herbicide) and on Th1 and Th2 cytokine secretion. Their possible protective role on intracellular stress oxidative induced by pesticides was also investigated. Peripheral blood lymphocytes were isolated using differential centrifugation on a density gradient of Histopaque. They were cultured with mitogen concanavalin A (Con A), Mancozeb and Metribuzin $(50 \mu \mathrm{M})$ in the presence or the absence of vitamin C $(50 \mu \mathrm{M})$ or NADH $(50 \mu \mathrm{M})$. Proliferation (MTT assay), IL-2, INF $\gamma$ and IL-4 (Elisa kits), oxidative markers (intracellular superoxide anion, hydroperoxides, carbonyl proteins, GSH, catalase and SOD) were determined. The results showed that pesticides were immunosuppressive and decreased cytokine secretion with a shift away from to Th1 phenotype. These immunomodulatory properties were accompanied by an increase in lymphocyte intracellular oxidative stress. The presence of vitamin $\mathrm{C}$ or $\mathrm{NADH}$, in the medium, exhibited protective effects in human lymphocytes by inhibiting pesticide-induced lymphocyte proliferation suppression, inflammatory status and oxidative stress generation. In conclusion, vitamin $\mathrm{C}$ and NADH can result in a safe and effective method to reduce pesticide adverse effects and help to restore immune function.
\end{abstract}

Key words: Antioxidants, cytokines, lymphocytes, Mancozeb, Metribuzin, NADH, oxidative stress, vitamin C.

\section{Introduction}

Pesticides are used in intensive agriculture worldwide. An important target organ of pesticide exposure is the immune system [1]. Lymphocyte proliferation assays and cytokine expression have been widely used for immunotoxicity assessment [2]. Ability of pesticides to stimulate or suppress lymphocyte proliferation and cytokine production, and to induce genotoxic activity and chromosomal aberrations in cultured lymphocytes was proposed as possible mechanisms explaining their adverse effects on immune system [3-6]. Cytokines, the important regulators of immune function play a crucial role in

Corresponding author: Merzouk Hafida, professor, research field: physiology and nutrition. activation, proliferation and differentiation of lymphocytes. Cytokine production was altered in response to pesticide exposure [7]. Several studies have also provided evidence for the involvement of reactive oxygen species (ROS) and oxidative stress in pesticide immunotoxicity $[8,9]$. ROS include the superoxide anion $\left(\mathrm{O}_{2}{ }^{-}\right)$, the hydroxyl radical ( $\mathrm{HO} \bullet$ ), and hydrogen peroxide $\left(\mathrm{H}_{2} \mathrm{O}_{2}\right)$. Oxidative stress has been strongly linked with the onset of various physical diseases. In the organism, enzymatic antioxidant defence systems including $\mathrm{NADH}$, quinone oxidoreductase 1, superoxide dismutase (SOD), catalase (CAT) and glutathione peroxidase (GPx) may neutralize the oxidative stress. Non-enzymatic antioxidants such as vitamin $\mathrm{E}$ and vitamin $\mathrm{C}$ can also act to overcome oxidative stress, being a part of the 
total antioxidant system. Vitamin $\mathrm{C}$ and vitamin $\mathrm{E}$ have been shown to possess anticarcinogenic, anticlastogenic, and antimutagenic properties in a variety of in vivo and in vitro models of pesticide exposure [10, 11]. Vitamin C (L-ascorbic acid) is hydrophilic and a very important free-radical scavenger in extracellular fluids, trapping radicals in the aqueous phase and protecting biomembranes from peroxidative damage. It prevents the increased production of free radicals induced by oxidative damage to lipids and lipoproteins in various cellular compartments and tissues [12]. Vitamin E is a lipid soluble, chain-breaking antioxidant playing a major protective role against oxidative stress and prevents the production of lipid peroxides by scavenging free radicals in biological membranes [12].

Nicotinamide adenine dinucleotide (NAD) and reduced nicotinamide adenine dinucleotide (NADH) have been known as classic molecules involving in energy metabolism, reductive biosynthesis, and antioxidation [13, 14]. Growing evidence has further indicated significant roles of NADH in important biological processes as aging, carcinogenesis, and immunological functions [15].

Evaluation of specific biochemical, molecular and immunologic events associated with $\mathrm{T}$ cell activation should become a routine analysis in the clinical laboratory in order to identify defects that result in impaired cellular immune functions. Quantifying $\mathrm{T}$ cell activation may become useful in monitoring the effect of immunomodulating therapies.

Mancozeb and Metribuzin are the most widely used pesticides in Algeria. Mancozeb is a dithiocarbamate fungicide used against fungal diseases of field crops and fruits. Metribuzin is a selective triazinone herbicide used mostly on vegetable crops. We have previously shown that these two pesticides had significant in vitro immunomodulatory properties with oxidative stress induction [16].

Therefore, in the current study we the possible protective role of vitamin $\mathrm{C}$ and $\mathrm{NADH}$ on intracellular stress oxidative induced by Mancozeb and Metribuzin. Lymphocytes were then treated with pesticides in the presence of vitamin $\mathrm{C}$ and NADH and the production of superoxide anion was measured as an indicator of oxidative stress.

\section{Material and Methods}

\subsection{Lymphocyte Proliferation Assay}

Peripheral blood was obtained from 10 healthy non-smoking male (mean age of 25 years) and 8 female (mean age of 25 years) donors, under no medication or food supplements intake and free of any known exposure to genotoxic agents. Fasting venous blood samples were collected in heparinized tubes. These samples were used for immediate lymphocyte isolation. The purpose of the study was explained to the volunteer subjects and their consent was obtained. The protocol was approved by the ethical committee of the Tlemcen-University Hospital.

Peripheral blood lymphocytes were isolated from heparinized venous blood using differential centrifugation ( $400 \mathrm{~g}$ for $40 \mathrm{~min}$ ) on a density gradient of Histopaque 1077 (Sigma). The peripheral blood lymphocytes at the interface of plasma and Histopaque were collected and washed twice with RPMI 1640 culture medium (Gibco, USA). After washing and counting, the cells were resuspended in RPMI medium at $4 \times 10^{6}$ cells $/ \mathrm{ml}$ concentration. For proliferation assay, $4 \times 10^{5}$ cells were cultured in triplicate in $200 \mu \mathrm{l}$ of medium RPMI 1640 supplemented with $25 \mathrm{mM}$ HEPES buffer, 10\% heat-inactivated fetal calf serum, L-glutamine $(2 \mathrm{mM})$, 2-mercaptoethanol $\left(5 \times 10^{-5} \mathrm{M}\right)$, penicillin $(100 \mathrm{UI} / \mathrm{ml})$ and streptomycin $(100 \mu \mathrm{g} / \mathrm{ml})$ with or without mitogen. Concanavalin A (Con A, Sigma, St Louis, MO, USA), a T-cell specific mitogen was used at $5 \mu \mathrm{g} / \mathrm{ml}$ final concentration. Cultures were grown in 96 flat-bottomed microtiter plates (Nunc, Paris, France) and maintained at $37{ }^{\circ} \mathrm{C}$ in a $5 \% \mathrm{CO}_{2}$ humidified atmosphere for $48 \mathrm{~h}$. In order to determine the effects of pesticides, lymphocytes were incubated with a 
concentration of $50 \mu \mathrm{M}$ of mancozeb and metribuzin initially dissolved in DMSO (final solvent concentration $<1 \%$ ). To determine the effects of vitamin $\mathrm{C}, 10 \mu \mathrm{M}$ of vitamin $\mathrm{C}$ and NADH (final concentration) were added in the culture medium. After incubation, cells were harvested by washing with RPMI 1640 medium. Cell viability was controlled by using a trypan blue exclusion test, and was unaffected by the compound concentrations used in our experiments (over 80\%). Proliferation was monitored by MTT [3- (4, 5-Dimethyl thiazol-2-yl)-2, 5-diphenyl tetrazolium bromide] (Sigma) assay. The absorbance of each sample and control (ConA-free medium) was read on a spectrophotometer at $565 \mathrm{~nm}$.

Stimulation index (PI) was calculated as follows:

SI $=$ (optical density of mitogen-stimulated cells / optical density of non-stimulated cells) $\times 100$.

\subsection{Interleukin-2, -4 and INFy Quantification}

Aliquots of culture supernatants were used to quantitate interleukins (IL-2, IL-4) and interferon- $\gamma$ (INF $\gamma$ ) by using commercially available ELISA kits (R \& D System, Oxford, UK), as per instructions furnished with. The results are expressed as $\mathrm{pg} / \mathrm{ml}$. The Th1/Th2 ratio was determined as the IFN $\gamma / \mathrm{IL}-4$ ratio.

\subsection{Lymphocyte Oxidant/Antioxidant Markers}

GSH measurement. Glutathione (GSH) levels were measured using a Bioxytech GSH-400 kit (OXIS International, Inc., Portland, OR, USA) as per the manufacturer's protocol. The absorbance at $400 \mathrm{~nm}$ was measured, and the GSH concentration was then determined with the GSH standard curve.

Determinations of lymphocyte antioxidant enzyme activities. After different incubations and cell collection, lymphocytes were washed once, resuspended in chilled $10 \mathrm{mM}$ phosphate buffer $(\mathrm{pH}$ 7.0) containing $0.15 \mathrm{M} \mathrm{NaCl}$, sonicated for $30 \mathrm{~s}$ and then centrifuged at $15,000 \mathrm{~g}$ for $20 \mathrm{~min}$. Aliquots of the supernatant fractions were used to determine the activities of catalase and the total activity of Superoxide dismutase ( $\mathrm{Cu}, \mathrm{Zn}-\mathrm{SOD}$ and $\mathrm{Mn}-\mathrm{SOD})$ according to established methods, as previously reported [16].

Determination of lymphocyte hydroperoxides. To determine markers of lipid peroxidation, hydroperoxides were measured, in sonicated lymphocyte supernatant, by the ferrous ion oxidation-xylenol orange assay (Fox 2) in conjunction with a specific $\mathrm{ROOH}$ reductant, triphenylphosphine (TPP) using a PeroxiDetect kit (Sigma, St. Louis, MO, USA) according to manufacture's instructions.

Determination of lymphocyte carbonyl proteins. Carbonyl proteins (markers of protein oxidation) were assayed in sonicated lymphocyte supernatant by the 2,4-Dinitrophenyl hydrazine reaction.

Lymphocyte superoxide anion determination: The generated superoxide anion $\left(\mathrm{O}_{2}{ }^{--}\right)$was quantified according to the method based on the reduction of nitroblue tetrazolium (NBT) to monoformazan by $\mathrm{O} 2^{\circ-}$. The yellow color of the reduced product was measured spectrophotometrically at $550 \mathrm{~nm}$.

\subsection{Statistical Analysis}

Data are expressed as mean \pm SD. Statistical analysis was carried out using STATISTICA, version 4.1 (Statsoft, Paris, France). Multiple comparisons were performed using ANOVA followed by the LSD (least significant difference) test. $\mathrm{P}<0.05$ was considered to represent significant statistical differences.

\section{Results}

\subsection{Lymphocyte Proliferation in the Presence of Pesticides and Antioxidants}

Pesticides (mancozeb and metribuzin) induced a significant reduction in the in vitro proliferative response of lymphocytes stimulated by mitogen con A as shown by stimulation index, SI (Table 1). However, lymphocyte proliferation stimulated with con A showed 
Table 1 Lymphocyte proliferation (stimulation index, SI) in the presence of pesticides (mancozeb and metribuzin) and antioxidants (Vitamin C, NADH).

\begin{tabular}{ll}
\hline SI Mancozeb & Human lymphocytes \\
\hline ConA & $207.48 \pm 20.63^{\mathrm{c}}$ \\
ConA+ Pest $50 \mu \mathrm{M}$ & $144.68 \pm 11.04^{\mathrm{d}}$ \\
ConA+ Vitamin C+ Pest $50 \mu \mathrm{M}$ & $280.59 \pm 10.11^{\mathrm{b}}$ \\
ConA+ Pest $50 \mu \mathrm{M}+\mathrm{NADH}$ & $356.45 \pm 16.15^{\mathrm{a}}$ \\
SI Metribuzin & \\
ConA & $207.48 \pm 20.63^{\mathrm{c}}$ \\
ConA+ Pest $50 \mu \mathrm{M}$ & $175.32 \pm 19.24^{\mathrm{d}}$ \\
ConA+ Vitamin C+ Pest $50 \mu \mathrm{M}$ & $296.49 \pm 20.88^{\mathrm{b}}$ \\
ConA+ Pest $50 \mu \mathrm{M}+\mathrm{NADH}$ & $360.23 \pm 22.16^{\mathrm{a}}$ \\
\hline
\end{tabular}

The values are means \pm SD of triplicate assays from 18 healthy subjects (10 men and 8 women). Multiple comparisons were performed using ANOVA followed by the least significant difference (LSD) test. a, b, c ... indicate significant differences obtained with different incubations $(\mathrm{P}<0.05)$.

a significant increase when treated with pesticides and antioxidants. The presence of vitamin $\mathrm{C}$ or NADH in the medium potentiated the stimulatory effects of con A upon lymphocyte proliferation. In fact, Vitamin C or $\mathrm{NADH}$ protected lymphocyte proliferation in the presence of pesticides. The effect was more pronounced with NADH in culture medium.

\subsection{Cytokine Secretion in the Presence of Pesticides and Antioxidants}

After incubations, the supernatant IL-2 and IFN $\gamma$ contents were measured as Th1 cytokine, while IL-4 was measured as Th2 cytokine (Table 2). Lymphocyte Th-1 (IL-2 and IFN $\gamma$ ) and Th-2 (IL-4) cytokine secretions were altered in the presence of Mancozeb and Metribuzin. IL-2, IFN $\gamma$ and IL-4 productions were significantly decreased by these pesticides. In addition, the Th1/Th2 ratio measured as the ratio IFN $\gamma / \mathrm{IL}-4$ was significantly increased indicating a pro-inflammatory status in the presence of pesticides.

The presence of vitamin $\mathrm{C}$ in the medium decreased IL-2 and IFN $\gamma$ secretions but increased IL-4 secretion and induced a reduction in the ratio IFN $\gamma / \mathrm{IL}-4$ indicating an anti-inflammatory effect.

The capacity of $\mathrm{T}$ cells to produce IL-4 was markedly enhanced after exposure to NADH, but IL-2 and INF $\gamma$ release did not change. The INF $\gamma / \mathrm{IL}-4$ ratio

Table 2 Lymphocyte Th1 ( IL-2, INF $\gamma$ ) and Th2 (IL-4) cytokine secretion in the presence of pesticides and antioxidants.

\begin{tabular}{|c|c|c|}
\hline & Mancozeb & Metribuzin \\
\hline & Human lymphocytes & Human lymphocytes \\
\hline \multicolumn{3}{|l|}{ IL-2 (Pg/mL) } \\
\hline ConA & $4129 \pm 119^{b}$ & $4129 \pm 119^{b}$ \\
\hline ConA + Pest $50 \mu \mathrm{M}$ & $1421 \pm 85^{\mathrm{d}}$ & $2521 \pm 104^{\mathrm{d}}$ \\
\hline ConA + vitamin $\mathrm{C}+$ Pest $50 \mu \mathrm{M}$ & $3356 \pm 127^{c}$ & $3221 \pm 144^{\mathrm{c}}$ \\
\hline ConA + Pest $50 \mu \mathrm{M}+\mathrm{NADH}$ & $5292 \pm 140^{\text {a }}$ & $5288 \pm 140^{\mathrm{a}}$ \\
\hline \multicolumn{3}{|l|}{$\mathrm{INF} \gamma(\mathrm{Pg} / \mathrm{mL})$} \\
\hline ConA & $540.42 \pm 30.18^{\mathrm{a}}$ & $540.42 \pm 30.18^{\mathrm{a}}$ \\
\hline ConA + Pest $50 \mu \mathrm{M}$ & $263.58 \pm 21.26^{\mathrm{c}}$ & $283.59 \pm 20.44^{\mathrm{b}}$ \\
\hline ConA + vitamin $\mathrm{C}+$ Pest $50 \mu \mathrm{M}$ & $315.04 \pm 19.55^{\mathrm{b}}$ & $302.33 \pm 18.50^{b}$ \\
\hline ConA + Pest $50 \mu \mathrm{M}+\mathrm{NADH}$ & $556.23 \pm 26.22^{\mathrm{a}}$ & $557.04 \pm 26.22^{\mathrm{a}}$ \\
\hline \multicolumn{3}{|l|}{ IL-4 (Pg/mL) } \\
\hline ConA & $58.37 \pm 4.16^{\mathrm{c}}$ & $58.37 \pm 4.16^{\mathrm{c}}$ \\
\hline ConA + Pest $50 \mu \mathrm{M}$ & $16.05 \pm 4.02^{\mathrm{d}}$ & $22.38 \pm 3.36^{\mathrm{d}}$ \\
\hline Con $\mathrm{A}+\operatorname{vitamin} \mathrm{C}+$ Pest $50 \mu \mathrm{M}$ & $70.27 \pm 3.78^{b}$ & $73.11 \pm 4.44^{\mathrm{b}}$ \\
\hline ConA + Pest $50 \mu \mathrm{M}+\mathrm{NADH}$ & $86.34 \pm 4.11^{\mathrm{a}}$ & $85.89 \pm 4.11^{\mathrm{a}}$ \\
\hline \multicolumn{3}{|l|}{$\mathrm{INF} \gamma / \mathrm{IL}-4$} \\
\hline ConA & $9.25 \pm 0.86^{\mathrm{b}}$ & $9.25 \pm 0.86^{\mathrm{b}}$ \\
\hline ConA + Pest $50 \mu \mathrm{M}$ & $16.20 \pm 1.04^{\mathrm{a}}$ & $12.86 \pm 1.63^{\mathrm{a}}$ \\
\hline Con $\mathrm{A}+\operatorname{vitamin} \mathrm{C}+$ Pest $50 \mu \mathrm{M}$ & $4.49 \pm 0.82^{\mathrm{d}}$ & $4.30 \pm 0.72^{\mathrm{d}}$ \\
\hline ConA + Pest $50 \mu \mathrm{M}+\mathrm{NADH}$ & $6.45 \pm 0.62^{\mathrm{c}}$ & $6.56 \pm 0.62^{\mathrm{c}}$ \\
\hline
\end{tabular}

The values are means \pm SD of triplicate assays from 18 healthy subjects (10 men and 8 women). Multiple comparisons were performed using ANOVA followed by the least significant difference (LSD) test. a, b, c ... indicate significant differences obtained with different incubations $(\mathrm{P}<0.05)$. 
Table 3 Cellular GSH, hydroperoxide, carbonyl protein and superoxide anion contents of stimulated $T$ lymphocytes in the presence of pesticides and antioxidants.

\begin{tabular}{lll}
\hline & Mancozeb & Metribuzin \\
\cline { 2 - 3 } & Human lymphocytes & Human lymphocytes \\
\hline CSH (nM/10 cells) & $12.89 \pm 1.14^{\mathrm{c}}$ & $12.89 \pm 1.14^{\mathrm{c}}$ \\
ConA + Pest $50 \mu \mathrm{M}$ & $7.22 \pm 1.05^{\mathrm{d}}$ & $8.33 \pm 1.11^{\mathrm{d}^{6}}$ \\
ConA + vitamin C+Pest $50 \mu \mathrm{M}$ & $15.76 \pm 1.27^{\mathrm{b}}$ & $18.21 \pm 1.33^{\mathrm{b}}$ \\
ConA + Pest $50 \mu \mathrm{M}+\mathrm{NADH}$ & $29.32 \pm 1.43^{\mathrm{a}}$ & $30.14 \pm 1.43^{\mathrm{a}}$ \\
HYDP(nM/10 cells) & & \\
ConA & $3.38 \pm 0.67^{\mathrm{b}}$ & $3.38 \pm 0.67^{\mathrm{b}}$ \\
ConA + Pest $50 \mu \mathrm{M}$ & $8.54 \pm 1.26^{\mathrm{a}}$ & $10.11 \pm 1.23^{\mathrm{a}}$ \\
ConA + vitamin C + Pest $50 \mu \mathrm{M}$ & $3.54 \pm 0.55^{\mathrm{b}}$ & $3.83 \pm 0.60^{\mathrm{b}}$ \\
ConA + Pest $50 \mu \mathrm{M}+\mathrm{NADH}$ & $2.62 \pm 0.52^{\mathrm{c}}$ & $2.54 \pm 0.52^{\mathrm{c}}$ \\
PCAR (nM/10 & & \\
ConA & $4.31 \pm 0.76^{\mathrm{b}}$ & $4.31 \pm 0.76^{\mathrm{b}}$ \\
ConA + Pest $50 \mu \mathrm{M}$ & $6.04 \pm 0.62^{\mathrm{a}}$ & $7.11 \pm 0.46^{\mathrm{a}}$ \\
ConA + vitamin C+Pest $50 \mu \mathrm{M}$ & $4.27 \pm 0.71^{\mathrm{b}}$ & $4.13 \pm 0.42^{\mathrm{b}}$ \\
ConA + Pest $50 \mu \mathrm{M}+\mathrm{NADH}$ & $4.22 \pm 0.51^{\mathrm{b}}$ & $4.30 \pm 0.51^{\mathrm{b}}$ \\
Superoxide anion (nM/10 & & \\
ConA & $19.25 \pm 1.86^{\mathrm{b}}$ & $19.25 \pm 1.86^{\mathrm{b}}$ \\
ConA + Pest $50 \mu \mathrm{M}$ & $36.20 \pm 2.04^{\mathrm{a}}$ & $28.86 \pm 1.63^{\mathrm{a}}$ \\
ConA + vitamin C + Pest $50 \mu \mathrm{M}$ & $22.49 \pm 2.82^{\mathrm{b}}$ & $21.30 \pm 2.72^{\mathrm{b}}$ \\
ConA + Pest $50 \mu \mathrm{M}+\mathrm{NADH}$ & $14.45 \pm 1.62^{\mathrm{c}}$ & $15.07 \pm 1.62^{\mathrm{c}}$ \\
\hline
\end{tabular}

The values are means \pm SD of triplicate assays from 18 healthy subjects (10 men and 8 women). Multiple comparisons were performed using ANOVA followed by the least significant difference (LSD) test. a, b, c ... indicate significant differences obtained with different incubations $(\mathrm{P}<0.05)$.

Table 4 Cellular antioxidant enzyme activities of stimulated $T$ lymphocytes in the presence of pesticides and antioxidants.

\begin{tabular}{lll}
\hline & Mancozeb & Metribuzin \\
\cline { 2 - 3 } & Human lymphocytes & Human lymphocytes \\
\hline Catalase (U/mg) & $22.89 \pm 1.13^{\mathrm{c}}$ & $22.89 \pm 1.13^{\mathrm{c}}$ \\
ConA & $47.76 \pm 1.54^{\mathrm{a}}$ & $48.17 \pm 1.34^{\mathrm{a}}$ \\
ConA + Pest $50 \mu \mathrm{M}$ & $35.38 \pm 1.27^{\mathrm{b}}$ & $30.29 \pm 1.32^{\mathrm{b}}$ \\
ConA + vitamin C + Pest $50 \mu \mathrm{M}$ & $23.26 \pm 1.22^{\mathrm{c}}$ & $21.41 \pm 1.22^{\mathrm{c}}$ \\
ConA + Pest $50 \mu \mathrm{M}+\mathrm{NADH}$ & & $93.33 \pm 1.67^{\mathrm{c}}$ \\
SOD (U/mg) & $93.33 \pm 1.67^{\mathrm{c}}$ & $100.26 \pm 1.34^{\mathrm{b}}$ \\
ConA & $99.02 \pm 1.26^{\mathrm{b}}$ & $123.33 \pm 2.63^{\mathrm{a}}$ \\
ConA + Pest $50 \mu \mathrm{M}$ & $114.14 \pm 2.55^{\mathrm{a}}$ & $91.33 \pm 1.52^{\mathrm{c}}$ \\
ConA + vitamin C + Pest $50 \mu \mathrm{M}$ & $92.24 \pm 1.52^{\mathrm{c}}$ & \\
ConA + Pest $50 \mu \mathrm{M}+\mathrm{NADH}$ & &
\end{tabular}

The values are means \pm SD of triplicate assays from 18 healthy subjects (10 men and 8 women). Multiple comparisons were performed using ANOVA followed by the least significant difference (LSD) test. a, b, c ... indicate significant differences obtained with different incubations $(\mathrm{P}<0.05)$.

was then reduced in the presence of NADH. However, the anti-inflammatory effect was more pronounced in the presence of vitamin $\mathrm{C}$.

\subsection{Lymphocyte Oxidative Stress Variables in the Presence of Pesticides and Antioxidants}

As shown in Table 3, pesticides induced an intracellular oxidative stress reflected by a reduction in glutathione content and an increase in superoxide anion, hydroperoxide and carbonyl protein contents in lymphocytes. Antioxidant enzyme activities were also altered by pesticides (Table 4), marked by an increase in catalase and SOD activities.

The addition of vitamin $\mathrm{C}$ and $\mathrm{NADH}$ to culture 
medium restored all these abnormalities. In fact, in the presence of vitamin $\mathrm{C}$ and $\mathrm{NADH}$, glutathione content was increased while superoxide anion, hydroperoxide and carbonyl protein contents were decreased in lymphocytes exposed to pesticides (Table 3). In the presence of antioxidants, catalase activity in lymphocytes exposed to pesticides was less induced by vitamin $\mathrm{C}$ and was kept normal by NADH (Table 4). However, SOD activity was stimulated by vitamin $\mathrm{C}$ and unchanged by NADH in lymphocytes treated with pesticides.

\section{Discussion}

Our current study demonstrated that vitamin $\mathrm{C}$ and $\mathrm{NADH}$ modulate in vitro lymphocyte proliferation, cytokine secretion and intracellular oxidative stress in the presence of pesticides. Vitamin $\mathrm{C}$ and NADH exhibited protective effects in human lymphocytes by inhibiting pesticide-induced lymphocyte proliferation suppression, inflammatory status and superoxide anion and oxidative stress generation.

The lymphocyte transformation assay is an important tool to measure in vitro mitogen-induced lymphocyte proliferation. This assay offers the opportunity to evaluate an impaired cellular immune response. Pro- and anti-inflammatory cytokines are mediators of the immune system and play an important role in inflammation, acute phase response and disease progression of pathological processes. The lymphocyte transformation assay is based on mitogen stimulation of lymphocytes, and is accepted as a technique to evaluate lymphocyte function. Con A represents the most powerful mitogen for lymphocytes. In our study, the lymphocyte proliferation responses to Con A were affected by the compounds used, the effect being related to the presence of Mancozeb and Metribuzin. Both of pesticides reduced lymphocyte proliferation, in agreement with our previous study [16]. Similar changes were also reported in cell culture following exposure to others pesticides $[5,17]$. In our study, the effects of the two pesticides on in vitro cytokine production were concomitant to those on lymphocyte proliferation, in agreement with our previous study [16]. In fact, we have measured different cytokine produced by lymphocytes to assess Th1/Th2 functional properties of lymphocytes. Possible explanation for inhibitory effects of pesticides could be due to the reduction of IL-2 secretion by stimulated lymphocytes, knowing that IL-2 is required for the proliferation of $\mathrm{T}$ cells. The alterations in lymphocyte proliferation after pesticide exposure in the current study could be also attributed to the modification of lymphocyte phenotype proportions. We noted that while pesticides induced a decrease in IL-2 and IFN- $\gamma$ secretion within lymphocytes, they also induced a reduction in IL-4 secretion. Th1-like response was nevertheless dominant reflecting probably the pro-inflammatory effect of pesticides. One possible explanation for the predominance of Th1 over Th2 in pesticide cultures could be an increase in the rate of Th2 lymphocyte apoptosis relative to that of Th1, as we have previously suggested [16].

In this study, lymphocyte redox markers were consistent with the induction of an oxidative stress in the presence of pesticides. Lymphocyte GSH levels were significantly reduced while superoxide anion, hydroperoxide and carbonyl protein levels were increased after pesticide treatment resulting probably from overproduction of free radicals and disturbance of the scavenging system. In addition, the increase in catalase and SOD activities could be an adaptative response to oxidative stress following pesticide exposure. Several studies showed that chronic exposure to pesticides suppresses humoral immune response, which is associated with increased excess of free radical generation [7, 8, 16, 18].

In our study, the addition of antioxidants, vitamin $\mathrm{C}$ and NADH to culture medium protected lymphocytes against the adverse effects of pesticides. Vitamin $\mathrm{C}$ and NADH stimulated lymphocyte proliferation despite the presence of pesticides. Treatment with 
these antioxidants induced a significant increase in cytokine release by lymphocytes exposed to pesticides. However, the INF $\gamma / \mathrm{IL}-4$ ratio was reduced by vitamin $\mathrm{C}$ and by NADH in pesticide treated lymphocytes, in favor of a shift of Th1/Th2 balance toward the Th2 phenotype. Previous studies demonstrated that vitamin $\mathrm{C}$ enhanced proliferative responses of $\mathrm{T}$ lymphocytes with an anti-inflammatory effect $[19,20]$. The anti-inflammatory effect of NADH has also been documented [21].

We observed that in the presence of antioxidants, the intracellular redox status from pesticide treated lymphocytes was normalized to control non treated values. We suggested that vitamin $\mathrm{C}$ and $\mathrm{NADH}$ regulated $\mathrm{T}$ cell redox status during pesticide exposure. In fact, in the presence of vitamin $\mathrm{C}$ and $\mathrm{NADH}$, superoxide anion, hydroperoxide and carbonyl protein levels were reduced while GSH contents and catalase activities were enhanced in pesticide treated lymphocytes. Previous findings had reported that vitamin $\mathrm{C}$ increased lymphocyte proliferation, reduced oxidative damage in cultured human lymphocytes and protected against cell destruction [19, 20]. In our study, SOD activity was stimulated by vitamin $C$ in lymphocytes exposed to pesticides. An increase in SOD activity is related to enhanced protection against oxidative stress. NADH might be the key protecting agent against free radicals and acting as a direct antioxidant, as previously reported [22, 23].

In conclusion, vitamin $\mathrm{C}$ and NADH can help to restore lymphocyte function and reduce intracellular oxidative stress in response to pesticide exposure. These antioxidants can result in a safe and effective method to reduce pesticide adverse effects and help to restore immune function.

\section{Acknowledgments}

This work was supported by the Algerian Research Project (PNR, 2011) and by SANMO (Algerian Society of orthomolecular nutrition and medicine) protocol. The authors thank INRAA (Algeria) for providing pesticides (Mancozeb and Metribuzin). The authors thank SANMO team for their technical help concerning vitamin supplementation.

\section{References}

[1] Corsini, E., Liesivuori, J., Vergieva, T., Van Loveren, H., and Colosio, C. 2008. "Effects of Pesticide Exposure on the Human Immune System." Hum. Exp. Toxicol. 27: 671-80.

[2] Carfi, M., Gennari, A., Malerba,I., Corsini, E., Pallardy, M., Pieters, R., Van Loveren, H., Vohr, H. W., Hartung, T., and Gribaldo, L. 2007. "In Vitro Tests to Evaluate Immunotoxicity: A Preliminary Study.” Toxicology 229: 11-22.

[3] Rencuzogullari, E., and Topaktas, M. 2000. "Chromosomal Aberrations in Cultured Human Lymphocytes Treated with the Mixtures of Carbosulfan, Ethyl Carbamate and Ethyl Methanesulfonate." Cytologia 65: 83-92.

[4] Muniz, J. F., McCauley, L., Scherer, J., Lasarev, M., Koshy, M., Kow, Y. W., Nazar-Stewart, V., and Kisby, G. E. 2008. "Biomarkers of Oxidative Stress and DNA Damage in Agricultural Workers: A Pilot Study."Toxicol. Appl. Pharmacol. 227: 97-107.

[5] Bolognesi, C. 2003. "Genotoxicity of Pesticides: A Review of Human Biomonitoring Studies." Mutat. Res. 543: 251-72.

[6] Neishabouri, E. Z., Hassan, Z. M., and Ostad, S. N. 2004. "Humoral and Cellular Immunomodulation Induced by Propoxure in C57-bl/6 Mice." Iranian J. Pharma. Res. 3: 41-5.

[7] Corsini, E., Codecà, I., Mangiaratti, S., Birindelli, S., Minoia, C., Turci, R., Viviani, B., Facchi, A., Vitelli, N., Lucchi, L., Galli, C. L., Marinovich, M., and Colosio, C. 2005. "Immunomodulatory Effects of the Fungicide Mancozeb in Agricultural Workers." Toxicol. Appl. Pharmacol. 208: 178-85.

[8] Li, Q., and Kawada, T. 2006. "The Mechanism of Organophosphorus Pesticide-Induced Inhibition of Cytolytic Activity of Killer Cells." Cell. Mol. Immunol. 3: 171-8.

[9] Saulsbury, M. D., Heyliger, S. O., Wang, K., and Round, D. 2008. "Characterization of Chlorpyrifos-Induced Apoptosis in Placental Cells." Toxicology 244: 98-110.

[10] Hoda, Q., and Sinha, S. P. 1993. "Vitamin C-mediated Minimisation of Rogor-Induced Genotoxicity." Mutat. Res. 299: 29-36.

[11] Atessahin, A., Yilmaz, S., Karahan, I., Pirincci, I., and Tasdemir, B. 2005. "The Effects of Vitamin E and Selenium on Cypermethrin-Induced Oxidative Stress in Rats." Turkish J. Vet. Anim. Sci. 29: 385-91. 
[12] Sies, H., Stahl, W., and Sundquist, A. R. 1992. "Antioxidant Function of Vitamin: Vitamins C \& E, $\beta$-carotene and Other Carotenoids." Ann. N Y Acad. Sci. 669: 7-12.

[13] Belenky, P., Bogan, K. L., and Brenner, C. 2007. "NADH Metabolism in Health and Disease." Trends Biochem. Sci. 32: 12-9.

[14] Berger, F., Ramirez-Hernandez, M. H., and Ziegler, M. 2004. "The New Life of a Centenarian: Signalling Functions of NAD(P)." Trends Biochem. Sci. 29: 111-8.

[15] Ying, W. 2008. "NAD+/ NADH and NADP/NADPH in Cellular Functions and Cell Death: Regulation and Biological Consequences." Antiox. Redox Signaling 10: 2.

[16] Medjdoub, A., Merzouk, H., Merzouk, S. A., Chiali, F. Z., and Narce, M. 2011. "Effects of Mancozeb and Metribuzin on in Vitro Proliferative Responses and Oxidative Stress of Human and Rat Spleen Lymphocytes Stimulated by Mitogens." Pesticide Biochemistry and Physiology 101: 27-33.

[17] Mehta, G., Singh, S. P., Pandey, S. K., and Sharma, L. D. 2008. "Cytotoxic Response of Endosulfan and Chlorpyrifos Pesticides in Poultry Lymphocyte Culture." Toxicol. Intern. 15: 97-101.
[18] Calviello, G., Piccioni, E., Boninsegna, A., Tedesco, B., Maggiano, N., Serini, S., Wolf, F. I., and Palozza, P. 2006. "DNA Damage and Apoptosis Induction by the Pesticide Mancozeb in Rat Cells: Involvement of the Oxidative Mechanism." Toxicol. Appl. Pharmacol. 211: 87-96.

[19] Molina, N., Morandi, A. C., Bolin, A. P., and Otton, R. 2014. "Comparative Effect of Fucoxanthin and Vitamin $\mathrm{C}$ on Oxidative and Functional Parameters of Human Lymphocytes." International Immunopharmacology 22: 41-50.

[20] Ströhle, A., and Hahn, A. 2009. "Vitamin C and Immune Function.” Med. Monatsschr. Pharm. 32: 49-54.

[21] Ungerstedt, J. S., Blomback, M., and Soderstom, T. 2003. "Nicotinamide Is A Potent Inhibitor of Proin Flammatory Cytokines." Clin. Exp. Immunol. 131: 48-52.

[22] Olek, R. A., Ziolkowski, W., Kaczor, J. J., Greci, L., Popinigis, J., and Antosiewicz, J. 2004. "Antioxidant Activity of NADH and Its Analogue- An In Vitro Study." J. Biochemistry Molecular Biology 37: 416-21.

[23] Cavallini, L., Valente, M., and Bindoli, A. 1983. "NADH and NADPH Inhibit Lipid Peroxidation Promoted by Hydroperoxides in Rat Liver Microsomes.” Biochimica Biophysics Acta 152: 339-45. 